\title{
EFFECTIVE MANAGEMENT OF CHRONIC CUTANEOUS ULCERS AS NON-CRITERIA MANIFESTATION OF ANTIPHOSPHOLIPID SYNDROME
}

Juliana Martins Brito ${ }^{1, \star}$, Paula Drumond Batista de Oliveira ${ }^{1}$, Farley Carvalho Araújo ${ }^{1}$

1.Universidade Federal dos Vales do Jequitinhonha e Mucuri, Diamantina (MG), Brazil.

*Corresponding author: juliana.martins.brito@hotmail.com

\section{BACKGROUND}

The antiphospholipid syndrome (APS) is a clinical condition characterized by thrombotic and/or obstetric events in patients with anticardiolipin antibodies, anti- $\beta 2$-glycoprotein I antibodies and/or lupus anticoagulant in at least two dosages within the interval of 12 weeks. This autoimmune condition can affect many systems of the body and the signs and symptoms will match the location where the events occurred. The manifestation can be neurological, cardiac, pulmonary, renal, hematological and dermatological. It is observed that deep venous thrombosis in lower limbs and fetal losses are the most typical manifestations of the disease, with few reports of isolated dermatological manifestations in case of APS. Dermatological manifestations associated with APS are livedo reticularis, lower limbs ulcers, subungual hemorrhages, digital gangrene, primary anetoderma, livedoid vasculitis and superficial thrombophlebitis.

\section{CASE REPORT}

A 61-year-old woman, three successful pregnancies, no abortion, menopause at 48, breast cancer treated for 4 years, showing no signs of recurrence, reported the appearance of painful ulcerated lesions for 3 years in the distal portion of the lower limbs. On the left, it extends from the medial malleolus to the lateral malleolus by posterior irradiation; on the right, it is located on the topography of soleus veins, both filled by granulation associated with healthy tissue, without signs of necrosis or infection, as shown in Fig. 1. She presented IgM anticardiolipin with moderate reactivity, confirmed after 12 weeks, negative IgG anticardiolipin, aPTT: 1.07, dilute Russell viper venom time (dRVVT) LA1: 1.39 and LA2: 1.09. Protein electrophoresis revealing polyclonal enlargement of the gamma globulin region, in addition to venous duplex scan of lower limbs excluding signs of thrombosis. Skin biopsy revealed the absence of necrosis or infectious signs. Nonreacting PPD, normal complete blood count, nonreactive Hep2, SSA/RO, HCV, HBSAg, HBC and rheumatoid factor, normal C3 and C4, fasting glucose level at $95.2 \mathrm{mg} / \mathrm{dL}$. Full anticoagulation was started with enoxaparin sodium $60 \mathrm{mg}$ SC twice a day and warfarin sodium $5 \mathrm{mg}$ once a day, with monitoring from INR; in 2 months, it showed significant improvement (Fig. 2). The Free Informed Consent Form was signed by the patient.

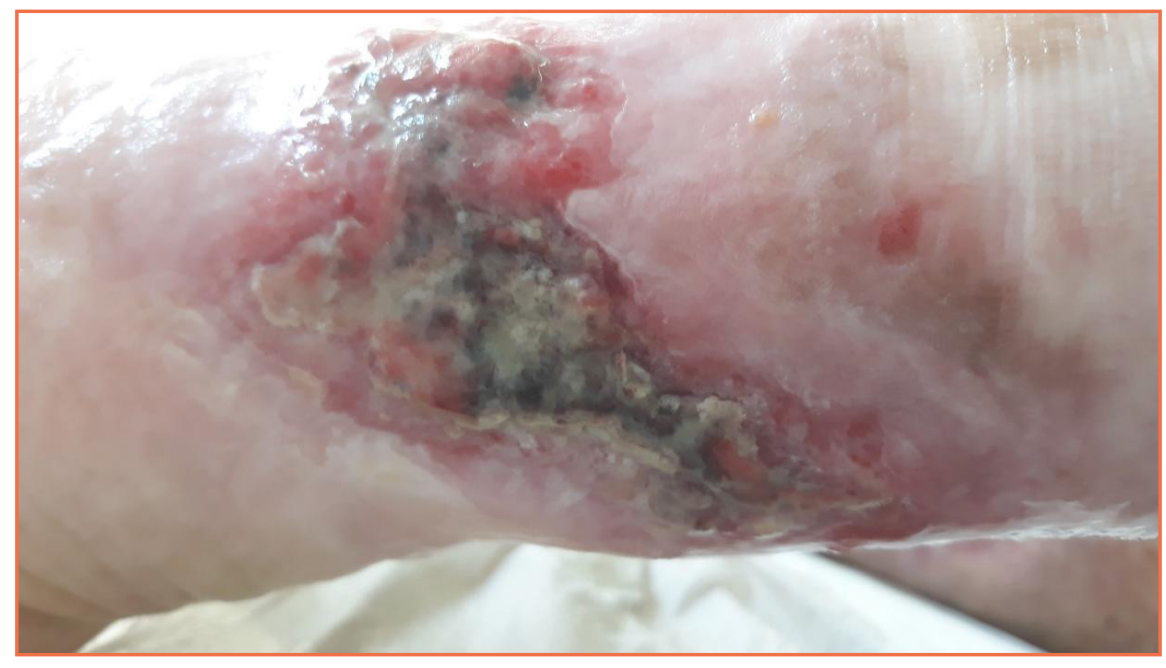

Figure 1. Ulcer in left lower limb before treatment. 


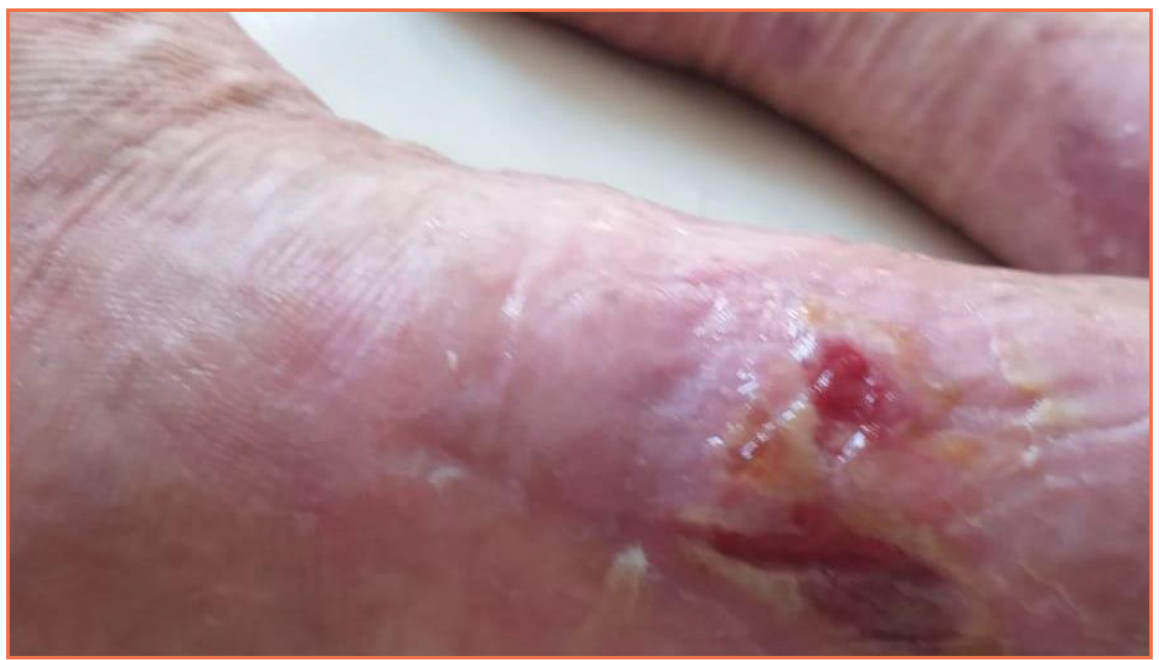

Figure 2. Wound in left lower limb after treatment.

\section{CONCLUSION}

Cutaneous ulcers resulting from APS, present in less than $10 \%$ of cases, are included as noncriteria manifestation. Despite this patient not matching the Sapporo's criteria, the ulcer showed improvement after treatment with anticoagulant. It is important to note that other etiologies were investigated and excluded. Therefore, even though an isolated cutaneous manifestation is rare, clinicians must keep the syndrome in mind and adapt the treatment of patients when necessary. 